\title{
The effect of heavy element opacity on pre-main sequence Li depletion
}

\author{
P. Sestito ${ }^{1,2}$, S. Degl'Innocenti ${ }^{3,4}$, P. G. Prada Moroni ${ }^{3,4}$, and S. Randich ${ }^{2}$ \\ 1 INAF/Osservatorio Astronomico di Palermo "Giuseppe S. Vaiana”, Piazza del Parlamento 1, 90134 Palermo, Italy \\ e-mail: sestito@arcetri.astro.it \\ 2 INAF/Osservatorio Astrofisico di Arcetri, L.go E. Fermi 5, 50125 Firenze, Italy \\ Dipartimento di Fisica “E. Fermi”, L.go B. Pontecorvo 2, 56127 Pisa, Italy \\ 4 INFN, Sezione di Pisa, L.go B. Pontecorvo 2, 56127 Pisa, Italy
}

Received 29 November 2005 / Accepted 21 March 2006

\section{ABSTRACT}

\begin{abstract}
Context. Recent 3-D analysis of the solar spectrum data suggests a significant change of the solar chemical composition. This may affect the temporal evolution of the surface abundance of light elements since the extension of the convective envelope is largely affected by the internal opacity value.

Aims. We analyse the influence of the adopted solar mixture on the opacity in the convective envelope of pre-main sequence (PMS) stars and thus on PMS lithium depletion. The surface Li abundance depends on the relative efficiency of several processes, some of them still not known with the required precision; this paper thus analyses one of the aspects of this "puzzle".

Methods. Focusing on PMS evolution, where the largest amount of Li burning occurs, we computed stellar models for three selected masses $\left(0.8,1.0\right.$ and $1.2 M_{\odot}$, with $\left.Z=0.013, Y=0.27, \alpha=1.9\right)$ by varying the chemical mixture, that is the internal element distribution in $Z$. We analysed the contribution of the single elements to the opacity at the temperatures and densities of interest for Li depletion. Several mixtures were obtained by varying the abundance of the most important elements one at a time; we then calculated the corresponding PMS Li abundance evolution.

Results. We found that a mixture variation does change the $\mathrm{Li}$ abundance: at fixed total metallicity, the Li depletion increases when increasing the fraction of elements heavier than $\mathrm{O}$.
\end{abstract}

Key words. stars: abundances - stars: evolution - stars: interiors

\section{Introduction}

In the last twenty years several observational studies of lithium $\left({ }^{7} \mathrm{Li}\right)$ in stars have been performed, many of which focus on open cluster objects in the zero-age main sequence (ZAMS) and in the main sequence (MS) phase (see the reviews by Jeffries 2000; Randich 2006; and the references quoted in Table 1 of Sestito \& Randich 2005). The temporal evolution of the surface Li abundance has also been investigated theoretically (e.g. D'Antona \& Mazzitelli 1997; review by Pinsonneault 1997, and references therein; Siess et al. 2000). The interest in Li depletion in stars comes from the fact that, due to its relatively low burning temperature $\left(\sim 2.5 \times 10^{6} \mathrm{~K}\right), \mathrm{Li}$ is a key tracer of the efficiency of mixing processes in stellar envelopes.

In spite of great efforts, the theory is still unable to reproduce the temporal behavior of surface $\mathrm{Li}$ abundances during the premain sequence (PMS) and MS phases, i.e. model predictions do not match the measured abundances for the Sun and for stars in open clusters of different ages (see, e.g. Jeffries 2000, and references therein).

The task is not easy because the temporal evolution of the surface chemical abundance (when the accretion phase has ended) depends on the relative efficiency of several processes: mixing, microscopic diffusion, radiative levitation and mass loss. In particular, the abundances of the light elements, which are easily burned, are extremely sensitive to the extent of envelope mixing. There are two main sources of uncertainties in these processes: the first one related to macroscopic processes and the other to microscopic physical ingredients adopted. Regarding the former, it is well known that due to the lack of a selfconsistent treatment of convection, stellar modelers are compelled to use relatively crude approximations depending on free parameters, such as the mixing length formalism. Even a precise physical treatment of the efficiency of the mass loss is not available. The situation is even worse if the assumption of spherical symmetry is relaxed allowing for rotation, or if magnetic fields are present. Once a scheme has been chosen for the treatment of a given macroscopic process (i.e. convective mixing), the prediction of its efficiency is still affected by uncertainties in the basic physical inputs such as the nuclear reaction rates, the equation of state and the opacity of the stellar matter (see e.g., D’Antona \& Mazzitelli 1997; Pinsonneault 1997; Turcotte \& Christensen-Dalsgaard 1998; Palla 2000; Jeffries 2006, for some results of evolutionary models). The canonical scenario assumes spherical symmetry with constant mass and adopts the classical Schwarzschild criterion for the identification of convective boundaries.

A fundamental role in $\mathrm{Li}$ evolution is played by uncertainties in the radiative opacity (which affects the depth of the convective envelope) and thus by the metal content, both the global abundance (i.e. the metallicity) and the distribution of the elements in the mixture. Recent analysis of solar spectroscopic data using 3-dimensional hydrodynamic atmospheric models (see Asplund et al. 2004, 2005 - hereafter As04, As05 - and references therein) have reduced the derived abundances of $\mathrm{CNO}$ 
and other heavy elements with respect to previous estimates (Grevesse \& Sauval 1998, hereafter GS98). GS98 improved the mixture by Grevesse \& Noels (1993, hereafter GN93), widely adopted in the literature, revising the $\mathrm{CNO}$ and $\mathrm{Ne}$ abundance and confirming the very good agreement between the new photospheric and meteoric results for iron. The $Z / X$ solar value decreased from the GN93 value $(Z / X)_{\odot}=0.0245$ to the GS98 estimate $(Z / X)_{\odot}=0.0230$ and then to the values $(Z / X)_{\odot}=0.0176$, 0.0165 found by As04 and As05, respectively. The corresponding solar metallicity values (i.e. assuming $[\mathrm{Fe} / \mathrm{H}]=0$, see also Sect. 5) for the different mixtures are $Z \sim 0.018$ (GN93 and GS98), $Z \sim 0.013$ (As04) and $Z \sim 0.012$ (As05). However, the problem of a precise determination of the solar mixture is still affected by theoretical and observational uncertainties and the quoted error on the $Z / X$ solar value is still about $10 \%$ (see e.g. Bahcall \& Serenelli 2005).

In this paper we analyse the influence of the adopted solar mixture on the opacity at the base of the convective envelope of pre-main sequence stars and thus on $\mathrm{Li}$ abundance in the convective envelope. We focus on PMS since, according to classical models, the largest amount of surface Li destruction for low mass stars occurs during this stage. The results are understood in terms of the contribution of each element to the total opacity at the physical conditions typical of the bottom of the convective zone during PMS. Clearly this is not an attempt to solve the "Li problem", which is much more complex, but a quantitative investigation of one of the aspects of the "puzzle".

A few studies have already been carried out concerning the dependence of Li depletion on element abundances and/or on the surface stellar opacities for solar-type stars (see e.g. Swenson et al. 1994a,b, and references therein; Piau \& Turck-Chièze 2002; Piau et al. 2003). Swenson et al. explored the effect of the opacity values (and of other physical inputs) on PMS Li depletion, as well as the effects of a variation of the oxygen abundance, trying to match the $\mathrm{Li}$ abundances observed for the Sun and the Hyades (under the assumption of no MS depletion), finding that an enhancement in $[\mathrm{O} / \mathrm{Fe}]$ leads to a larger Li destruction. They also noted that heavier species such as $\mathrm{Fe}, \mathrm{Ne}, \mathrm{Mg}$ and Si might significantly contribute to the opacity.

Similarly, Piau \& Turck-Chièze (2002) presented PMS models with Pleiades (solar) and Hyades-like compositions. They investigated the dependence of Li depletion on metal and He fractions, by varying the global mixture (and changing the total $Z$ ); they showed that $\mathrm{Li}$ abundances are very sensitive to composition, suggesting that the dispersion in the observed values in open clusters could be related to small changes in the metal mixture among the cluster stars.

Piau et al. (2003) investigated the sensitivity of the extension of the convective zone during the MS for a mass of $\sim 1 M_{\odot}$, with respect to the surface content of CNO. Their models are calculated by changing $[\mathrm{CNO} / \mathrm{Fe}]$ while maintaining $[\mathrm{Fe} / \mathrm{H}]$ and the effective temperature unaltered. Thus, the total $Z$ increases if CNO increases (and vice versa). Under these conditions, Piau et al. found that an enhancement of CNO results into a smaller amount of Li depletion. Piau (2005) investigated the history of $\mathrm{Li}$ isotopes in Population II dwarfs, finding a correlation between the scatter in $\mathrm{Li}$ abundance and variations in $[\mathrm{Fe} / \mathrm{O}]$ ratios, i.e. changes in $[\mathrm{Fe} / \mathrm{O}]$ affect $\mathrm{Li}$ depletion more than a change in the global metallicity.

The paper is organized as follows: Sect. 2 briefly describes the evolutionary code and the physical inputs adopted for the calculations; in Sect. 3 we present the results of our computations for models with different mixtures and fixed metallicity $(Z=0.013)$, which are interpreted in Sect. 4. In Sect. 5 we also discuss the effect of changing both the mixture and the total metallicity value $Z$. A summary (Sect. 6) closes the paper.

\section{Evolutionary code and input physics}

The models have been computed with an updated version of the FRANEC evolutionary code (see e.g. Chieffi \& Straniero 1989); the physical inputs adopted for the calculations have been listed in the Appendix of Cariulo et al. (2004) - see also Ciacio et al. (1997). With respect to Cariulo et al. (2004) we updated the equation of state (OPAL EOS_2001) ${ }^{1}$, see also Rogers (2001), Iglesias \& Rogers (1996), and the conductive opacity (Potekhin 1999). The nuclear cross sections for Li burning are taken from the NACRE compilation (Angulo et al. 1999). The adopted initial value for $\mathrm{Li}$ in disk stars is $\log n(\mathrm{Li})=3.3^{2}$ (see, e.g. Jeffries 2006), or $N_{\mathrm{Li}} / N_{\mathrm{H}} \sim 2 \times 10^{-9}$.

The opacity values are fundamental for model computation; we adopt the radiative opacities by the Livermore group (Iglesias \& Rogers 1996) for temperatures higher than $12000 \mathrm{~K}$ and those by Alexander \& Ferguson (1994) for the lower temperatures. As it will be discussed in the next section, we used several different mixtures for the high temperature opacities, while the Grevesse \& Anders (1991) mixture has been adopted for the molecular atmospheric opacities. We also notice that the adopted EOS is calculated for the Grevesse \& Anders (1991) mixture; to our knowledge EOS tables with an updated mixture are not available, however, as stated in the OPAL EOS web site, these abundance changes should have very small effects on the EOS.

The classical Schwarzschild criterion has been adopted to determine the extension of the convective envelope and the efficiency of the energy transport has been modeled with mixing length formalism. We did not include rotation.

Element diffusion is included (Ciacio et al. 1997; with diffusion coefficients from Thoul et al. 1994), while radiative acceleration has been neglected since we analysed only the PMS phase of low mass stars $\left(M \leq 1.2 M_{\odot}\right)$. In fact, as discussed by Turcotte et al. (1998a,b), for stars with solar composition and masses around $1 M_{\odot}$ the effect of the radiative levitation becomes comparable to that of the gravitational settling only at the end of the MS phase.

In the following calculations, due to the uncertainties already discussed, it is not possible to perform precise evaluations of the absolute Li surface values; we will instead perform a "differential" analysis with respect to a "standard" model, investigating the effects of a mixture change. Our "standard" model adopts $[\mathrm{Fe} / \mathrm{H}]=0$ with the As04 mixture (corresponding to a metallicity of $Z \sim 0.013), Y=0.27$ and the mixing length parameter $(\alpha)$ equal to 1.9. The effects of a variation of $[\mathrm{Fe} / \mathrm{H}], Y$ and $\alpha$ within a plausible range of uncertainty will also be discussed.

\section{Li abundance for models with different mixtures}

\subsection{Reference models}

We investigated three models of different masses $(0.8,1.0$ and $\left.1.2 M_{\odot}\right)$, representing the typical mass range covered by most observational data for $\mathrm{Li}$ in open clusters; higher masses have very shallow convective envelopes and thus the effect we are studying is too small to be accurately investigated.

The various models discussed in the present paper are summarized in Table 1: Cols. 1 and 2 report the sequence number

\footnotetext{
1 http://www-phys.1lnl.gov/Research/OPAL

2 Adopting the usual scale: $\log n(\mathrm{Li})=12+\log \left(N_{\mathrm{Li}} / N_{\mathrm{H}}\right)$.
} 
Table 1. Summary of the models computed in this work. In all cases the total metallicity is fixed to $Z=0.013$, with the exception of model No. 16; the PMS evolution has been followed for the three masses $1.2,1.0$ and $0.8 M_{\odot}$.

\begin{tabular}{cccc}
\hline \hline No. & Label & Mixture & Element, opacity, or metallicity variations \\
\hline 1 & As04 (standard) & Asplund et al. (2004) & Standard solar mixture \\
2 & GN93 & Grevesse \& Noels (1993) & Standard solar mixture \\
3 & GS98 & Grevesse \& Sauval (1998) & Standard solar mixture \\
4 & As05 & Asplund et al. (2005) & Standard solar mixture \\
5 & As04 (atmospheric opacity decr. by 5\%) & Asplund et al. (2004) & Atmospheric opacity decreased by 5\% \\
6 & As04 (atmospheric opacity decr. by 10\%) & Asplund et al. (2004) & Atm. opac. decreased by 10\% \\
7 & Only C & - & Metallicity composed by 100\% C \\
8 & Only O & - & $100 \%$ O \\
9 & Only Ne & - & $100 \%$ Ne \\
10 & $\mathrm{C}(-20 \%) \mathrm{O}(+)$ & Asplund et al. (2004) & C mass fraction decreased by 20\% (compensated by O enhancement) \\
11 & $\mathrm{O}(-20 \%) \mathrm{C}(+)$ & Asplund et al. (2004) & O mass fraction decreased by 20\% (compensated by C enhancement) \\
12 & $\mathrm{Ne}(+30 \%) \mathrm{O}(-)$ & Asplund et al. (2004) & Ne mass fraction enhanced by 30\% (compensated by O decrease) \\
13 & $\mathrm{Ne}(-30 \%) \mathrm{O}(+)$ & Asplund et al. (2004) & Ne mass fraction decreased by 30\% (compensated by O enhancement) \\
14 & Heavy elements $(+20 \%)$ & Asplund et al. (2004) & Elements heavier than O increased by 20\% \\
15 & $\mathrm{CNO}(+20 \%)$ & Asplund et al. (2004) & CNO increased by 20\% \\
16 & GN93 $Z=0.018$ & Grevesse \& Noels (1993) & $Z$ \\
\hline
\end{tabular}

of the model and the label used in the figures, respectively; in Col. 3 the adopted solar mixture is given, while Col. 4 lists the variations of elements with respect to the solar mixture - if any - or the variations in the surface opacity or global metallicity.

The upper panel of Fig. 1 shows the evolutionary tracks for the three selected masses from the PMS to the bottom of the red giant branch (RGB) with our reference choice of metallicity and heavy element mixture (model No. 1, As04, in Table 1). Note that for completeness we describe here the evolution up to the RGB; however, the following discussions will focus only on the evolution up to the ZAMS phase where the largest amount of $\mathrm{Li}$ depletion occurs. As well known, the treatment of the early PMS phases is very problematic and the definition of the birth line requires the adoption of hydrodynamical codes (see e.g. Stahler \& Palla 2004, for an extensive analysis). However, this is not a crucial point for our purposes since the stellar characteristic after the birth line are largely insensitive to the previous evolutionary history. Moreover, for the selected masses the equilibrium is reached very early (ages $\lesssim 1 \mathrm{Myr}$ ), when they have not yet started surface Li depletion: indeed, at the beginning of the PMS phase, the zone where the Li burning temperature is exceeded represents a small fraction of the total external convective region; the dilution is thus very large. For these reasons we will show our calculations starting from an age of $1 \mathrm{Myr}$.

The middle panel of Fig. 1 shows the behavior of Li abundance $\left(N_{\mathrm{Li}} / N_{\mathrm{H}}\right)$ as a function of the age for the same models, while in the lower panel the temperature at the bottom of the convective zone $\left(T_{\mathrm{cz}}\right)$ is reported. As well known, the smaller the mass the deeper the convective zone and thus the higher the temperature at its lower edge. During the PMS the extension of the convective envelope and $T_{\mathrm{cz}}$ decrease until, in the cases of 1 and $1.2 M_{\odot}$, it becomes lower than $\sim 2.5 \times 10^{6} \mathrm{~K}$; as a consequence $\mathrm{Li}$ destruction stops and the surface abundance decreases only due to microscopic diffusion. On the contrary, for the model with $0.8 M_{\odot} \mathrm{Li}$ burning is efficient also during the MS phase even if the largest amount of $\mathrm{Li}$ is depleted during the PMS phase (note that MS Li depletion is not evident from the figure due to the large scale adopted: for instance, from the ZAMS to the turn off, $N_{\mathrm{Li}} / N_{\mathrm{H}}$ decreases from $\sim 6 \times 10^{-12}$ to $\sim 1.5 \times 10^{-13}$, i.e. by a factor $\sim 40$ ).

After central hydrogen exhaustion, the $\mathrm{H}$ burning continues in a shell surrounding the He core and the stars move to lower effective temperatures. During this phase the convective envelope extends into regions in which $\mathrm{Li}$ has accumulated due to the effect of diffusion, and the external abundance temporarily increases; then, it decreases below the level of detection when the external convective region reaches the layers in which $\mathrm{Li}$ has been completely burned (dilution effect).

The details of Li depletion depend on the adopted physical inputs, in particular the radiative opacity and chemical composition for a given mixing scheme. Thus, the situation shown in Fig. 1 has to be considered as a description of the dependence of Li depletion on the mass, while the detailed quantitative values hold only for the labeled parameters.

\subsection{Global uncertainties}

Empirical data and theoretical predictions are usually compared in the $\left[\log n(\mathrm{Li}), T_{\text {eff }}\right]$ plane; thus it is important to evaluate the ability of canonical models to reproduce these quantities. The present empirical uncertainties on the metallicity, He abundance and efficiency of the external convection (usually parameterized by the mixing length value $\alpha$ ) play an important role in the comparison between theory and observations.

At the typical effective temperatures of the stars under investigation the surface He abundance cannot be measured spectroscopically. As a consequence one has to rely on indirect methods, such as assuming a relation between metal and He enrichment of the Galactic medium which is not well defined (see, e.g., Pagel \& Portinari 1998; Castellani et al. 1999; Luridiana et al. 2003; Olive \& Skillman 2004). Under this assumption, $\Delta Y= \pm 0.02$ is generally assumed as an estimate of the original He abundance uncertainty for these stars.

Regarding the Fe content, disk stars cover a wide interval of $[\mathrm{Fe} / \mathrm{H}]$ and typical uncertainties depend on several factors such as on whether the analysis is spectroscopic or photometric, the quality of the data, the method of analysis, statistics etc. In the case of young nearby stars (see e.g. Chen et al. 2000; Bensby et al. 2003) and open clusters with nearly solar metallicity (e.g. Sestito et al. 2003; Carretta et al. 2004; Randich et al. 2006) the quoted errors range from $\Delta[\mathrm{Fe} / \mathrm{H}] \sim \pm 0.01$ up to $\sim \pm 0.06 \div 0.07$, when the analysis is based on a large sample of stars observed with high resolution spectrographs. Errors can be as high as $\Delta[\mathrm{Fe} / \mathrm{H}] \sim \pm 0.1$ (or more) if results from low resolution spectroscopy or from the photometry are considered (see e.g. Friel et al. 2002; Nordström et al. 2004). We adopt here a value of $\Delta[\mathrm{Fe} / \mathrm{H}]= \pm 0.1$ as a conservative estimate of observational uncertainties on the metallicity of solar-type stars. 

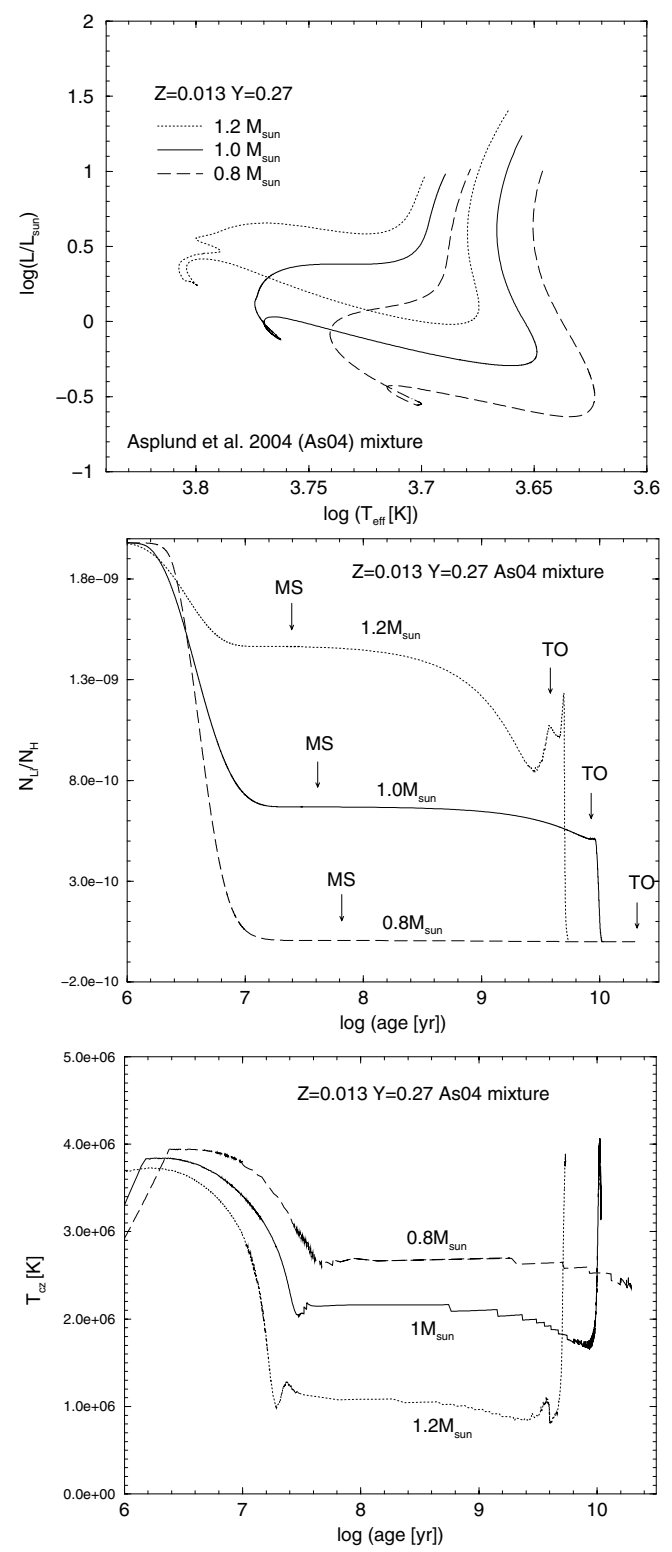

Fig. 1. Upper panel: the evolution in the HR diagram of our three selected "standard" models $\left(0.8,1.0\right.$ and $1.2 M_{\odot}$, with $Z=0.013$, $Y=0.27$ and $\alpha=1.9$; Asplund et al. $2004-$ As04 - mixture) from the PMS to the RGB phase. Middle panel: surface Li abundance as a function of time (log age) for the three models. The abundance is expressed as the ratio between $\mathrm{Li}$ and $\mathrm{H}$ numerical abundances $\left(N_{\mathrm{Li}} / N_{\mathrm{H}}\right)$; the starting of the MS phase and the turn off (TO) positions are indicated. Lower panel: temperature at the bottom of the convective envelope $\left(T_{\mathrm{cz}}\right)$ vs. log age (when the star is fully convective the bottom of the convective core coincides with the center of the star); the indicative temperature of ignition for the ${ }^{7} \mathrm{Li}+\mathrm{p}$ reaction is $\sim 2.5 \times 10^{6} \mathrm{~K}$.

Finally, the $\alpha$ parameter adopted in the calculations, depending on the selected set of atmospheric models, could be calibrated by requiring the reproduction of the color of the MS stars of the observed open cluster. However the parameter $\alpha$ is used to take into account our lack of precise knowledge about the convection mechanisms, and there is no reason for which it must assume the same value for stars of different mass and chemical composition, or even for different evolutionary phases of the same star. When a direct calibration of the $\alpha$ value for PMS stars through the reproduction of their stellar colors is not possible,

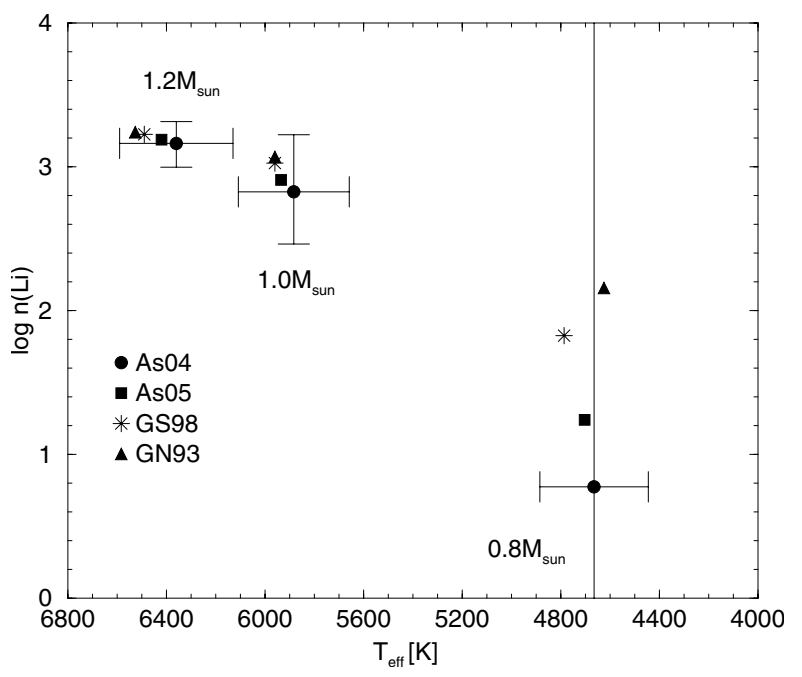

Fig. 2. Theoretical Li abundance (expressed as $\log n(\mathrm{Li})=12+$ $\left.\log \left(N_{\mathrm{Li}} / N_{\mathrm{H}}\right)\right)$ as a function of the effective temperature for $0.8,1.0$ and 1.2 $M_{\odot}$ at the age of $\sim 30$ Myr. Results for the four labeled different mixtures are shown (Grevesse \& Noels 1993 - GN93; Grevesse \& Sauval 1998 - GS98; Asplund et al. 2004 - As04, our "standard" mixture; Asplund et al. 2005 - As05). For As04 we also show the error bars due to the estimated uncertainties on $[\mathrm{Fe} / \mathrm{H}], Y$ and $\alpha$ (see text).

one must take into account an uncertainty on this parameter; we allow a variation of the mixing length parameter $\alpha$ by \pm 0.3 .

We computed six models for each of the three selected masses by fixing, in turn, one of the three parameters at its boundary values (lower and upper) and keeping the others at their central values $(Z=0.013, Y=0.27, \alpha=1.9)$. In this way, we obtained relative variations of $\mathrm{Li}$ abundance and effective temperature with respect to our "standard" model, representing an evaluation of the uncertainties on $\mathrm{Li}$ and $T_{\text {eff }}$ due to plausible empirical errors on $Z, Y$ and $\alpha$. Then, the relative variations of $\mathrm{Li}$ and $T_{\text {eff }}$ (on the increase and on the decrease) due to each of the quoted parameters are added quadratically to give a quantitative estimate of the total (upper and lower) uncertainties.

In Fig. 2 the results for our "standard" models for the three different masses at $30 \mathrm{Myr}$ (when for all the stars the main Li depletion has ended) are shown in the $\left[\log n(\mathrm{Li}), T_{\text {eff }}\right]$ plane with the error bars due to the ranges of $[\mathrm{Fe} / \mathrm{H}], Y$ and $\alpha$, calculated as described above. The error bars represent the range of predicted theoretical values due to the uncertainties discussed above, while intrinsic errors of the model (due to physical inputs) are not considered; therefore the error bars are an indicative estimate of the uncertainties in the initial physical parameters. Note that among the three quoted error sources, the largest contribution to the total error bar is due to the uncertainty on $Z$; in particular, the effect of the metallicity variation is very strong for the lowest mass, for which $\log n(\mathrm{Li})$ appears to be unpredictable.

\subsection{Heavy element mixture variations and opacity effect}

The next step is aimed to evaluate the effect of a variation of the adopted heavy element mixture with respect to the sources of uncertainty described above. Thus we calculated models for the same $(Z, Y, \alpha)$ and the Grevesse \& Noels (1993), Grevesse $\&$ Sauval (1998) and Asplund et al. (2005) mixtures (models Nos. 2, 3, 4 in Table 1; GN93; GS98; As05). Since the temperatures of main interest for $\mathrm{Li}$ depletion are those at the bottom of the external convective region during PMS Li burning, that is $T_{\mathrm{cz}} \sim 3 \div 4 \times 10^{6} \mathrm{~K}$ (see Fig. 1), we calculated high temperature 

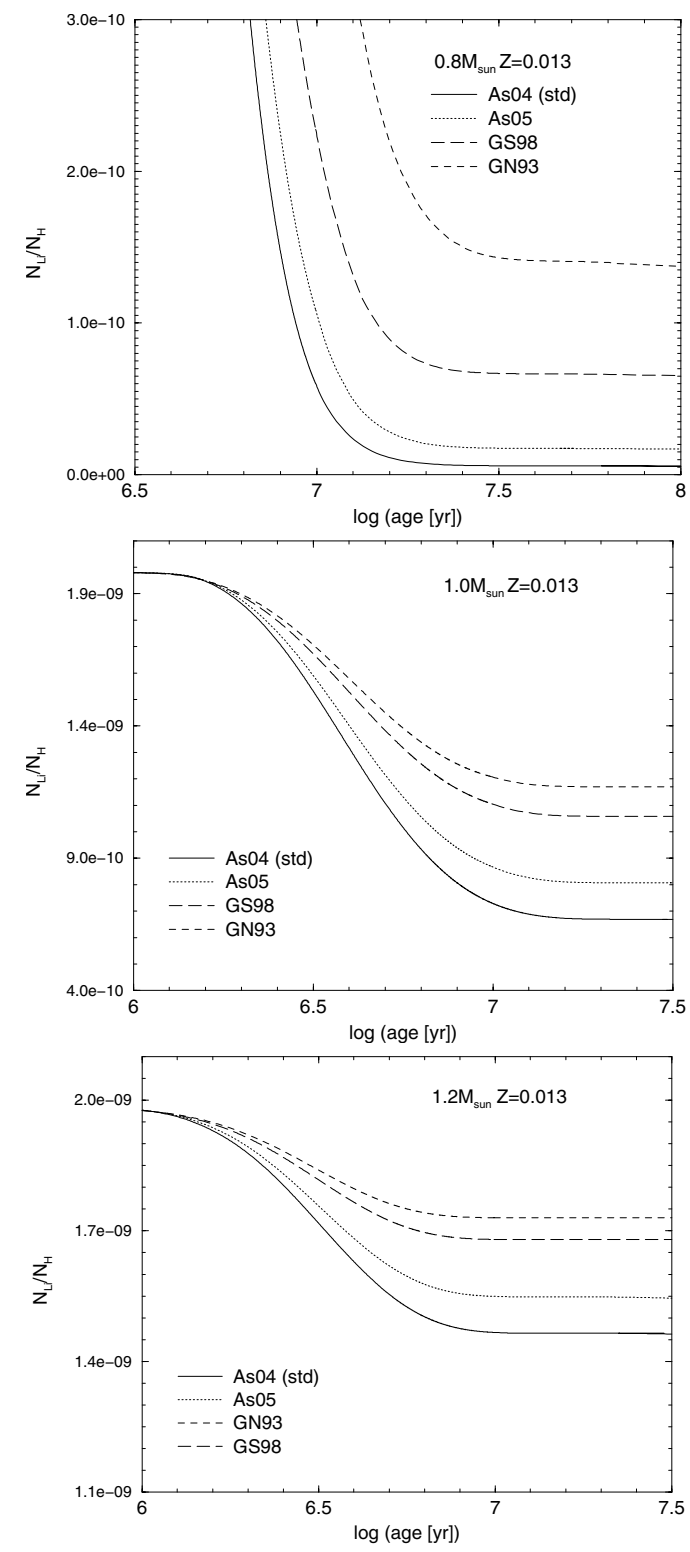

Fig. 3. Surface Li abundance behavior during the PMS phase for the different labeled mixtures. The three selected masses are reported: $0.8 M_{\odot}$ (upper panel), $1.0 M_{\odot}$ (middle panel), $1.2 M_{\odot}$ (lower panel), for $Z=0.013, Y=0.27, \alpha=1.9$. Abundances and ages are expressed as in Fig. 1; note the different scales for Li abundances and ages adopted in the three panels.

opacities $(T>12000 \mathrm{~K})$ for the chosen mixtures using the procedures available on-line from the OPAL group.

The results of our calculations are reported in Fig. 2. The variations in $\mathrm{Li}$ abundance and $T_{\text {eff }}$ due to the mixture changes are within the (conservative) previously quoted uncertainties but they are not negligible. It can also be noticed that the smaller the mass, the more sensitive is the dependence of the Li abundance on the adopted physical parameters.

For completeness Fig. 3 shows the surface Li abundance behavior during the PMS phase for the three selected masses with the four different mixtures of Fig. 2. The models that undergo the largest amount of Li depletion have higher opacity in the region at the bottom of the convective zone, and thus deeper and hotter bottom convective envelopes (see Sect. 4).

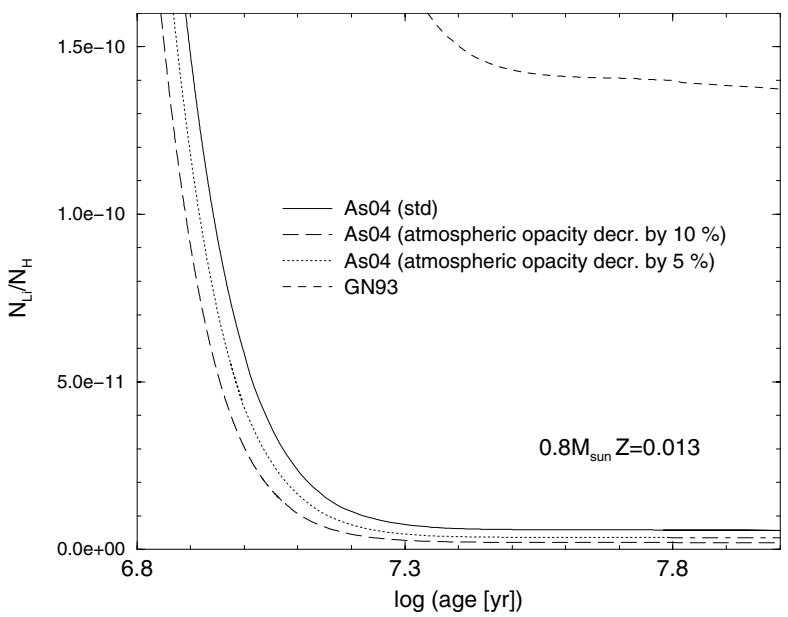

Fig. 4. Surface Li abundance vs. age for $0.8 M_{\odot}(Z=0.013, Y=0.27$, $\alpha=1.9$ ). The standard As04 model is compared to models in which the atmospheric opacity has been decreased by $5 \%$ (dotted line) and by $10 \%$ (long dashed line). We report for reference also the model with the mixture by GN93.

However, the various quoted mixtures differ in the abundance of many elements. In the following (Sect. 4), we will thus discuss simpler situations in which only one or a few critical element abundances are changed. This provides a better understanding of the dependence of the opacity at the bottom of the convective envelope - and thus of Li depletion - on the element abundance variations.

Low temperature (i.e. atmospheric) opacities calculated for the As04 and As05 mixtures are not available; however, since the temperature of the bottom of the convective zone is relatively high, the effect of a change in the surface opacity due to the mixture update on Li depletion should not be too relevant. We expect that changes in atmospheric opacities mostly affect the model with the smallest mass $\left(0.8 M_{\odot}\right)$, which has the lowest effective temperature and the deepest convective envelope. From the calculations by the OPAL group we found that, for a $0.8 M_{\odot}$ star and at a temperature of about $12000 \mathrm{~K}$ (the lowest $T$ for which the OPAL tables are computed), the change in the internal opacity values when going from the GN93 to the As04 mixture (at fixed $Z$ ) is less than 5\%; we took this value as representative also of a possible variation in the lower temperature opacities. In order to analyse the effect of such a variation, we evolved a $0.8 M_{\odot}$ ( $Z=0.013, Y=0.27, \alpha=1.9$ ) with the usual atmospheric opacities (Alexander \& Ferguson 1994) artificially decreased by 5 and $10 \%$ (models Nos. 5 and 6 in Table 1). For the interior opacities we adopted the As04 mixture. The results for Li depletion are shown in Fig. 4 compared with the "standard" $0.8 M_{\odot}$. The effect is rather small; the decreased atmospheric opacities result in models with hotter external regions, thus, even if the depth of the convective envelope is nearly unchanged, their bottom temperature is slightly higher and a slightly larger amount of Li depletion occurs. Nevertheless, the effect is negligible with respect to that due to a mixture variation for the interior: the ZAMS Li abundances (age $\sim 30 \mathrm{Myr}$ ) decrease by factors $\sim 1.5$ and 3 (with respect to the As04 standard model) when surface opacities are decreased by 5 and $10 \%$, respectively; on the other hand, going from the standard As04 mixture to the GN93 one, the ZAMS Li content increases by a factor $\sim 18$. 


\section{Interpretation of the $\mathrm{Li}$ abundance behavior}

In order to understand the effect of the different mixtures on the extension of the convective envelope, we analyse the contribution of the different elements to the radiative opacity at the temperatures of interest, i.e. those at the base of the convective envelope during the PMS. Therefore, we computed opacity tables for pure elements by using the data and routines available on-line form the LAOL group ${ }^{3}$ (we did not use OPAL opacities in this case because they are not available for a single element).

Figure 5 shows the pure element opacity for the most important elements: $\mathrm{H}, \mathrm{He}, \mathrm{C}, \mathrm{O}, \mathrm{Ne}, \mathrm{Fe}, \mathrm{Si}$. The opacities are calculated for $T=3.5 \times 10^{6} \mathrm{~K}$ and $T=4.6 \times 10^{6} \mathrm{~K}$ and for densities ranging from 0 to $10 \mathrm{~g} \mathrm{~cm}^{-3}$. Note that at the selected temperatures the most opaque element is $\mathrm{Fe}$, followed by $\mathrm{Ne}$, and $\mathrm{O}$ is more opaque than $\mathrm{C}$; the opacity of pure $\mathrm{He}$ and $\mathrm{H}$ is negligible with respect to those of metals. Obviously in a realistic case these single element opacities must be weighted with the abundance of each element in a mixture in which $\mathrm{H}$ and $\mathrm{He}$ are present too.

The next step to understand the results of Sect. 3 is thus to compute PMS models in which, for the standard metal and He abundances, the contribution to the metallicity is made by only one element. Therefore, we evolved the models for $Z=0.013$ and $Y=0.27$ by assuming that the contribution to the metallicity is made by only $\mathrm{C}, \mathrm{O}$ or $\mathrm{Ne}$; we also took into account the variation of the composition in calculating the nuclear reaction rates, while for the other thermodynamical quantities we adopted the OPAL EOS_2001 tables calculated for the Grevesse (1991) solar mixture. Our assumptions, as a first approximation, affect the PMS evolution only through the variation of the stellar opacity; we checked that the temperature profile in the convective envelope does not change in a significant way for the three models.

The opacity tables were computed by adopting the procedures available on-line from the OPAL group. The results of these numerical experiments are shown in Fig. 6 (models Nos. 7, 8, 9 of Table 1); the behavior of $\mathrm{Li}$ abundance is easily explained by the opacity values seen in Fig. 5: the element with the highest opacity at temperatures and densities typical of the base of the convective envelope leads to the highest Li depletion for the models of Fig. 6. In addition, the results for our "standard" models are not so different from those obtained for a metallicity contributed by only $\mathrm{O}$ : this is easily understandable, since $\mathrm{O}$ is the most abundant element (after $\mathrm{H}$ and $\mathrm{He}$ ) and its opacity is greater than that of $\mathrm{C}$ (which is the next most abundant element). The behavior is similar for the three masses although, as already noted in Fig. 3, the effect is smaller for larger stellar masses.

A more realistic situation is represented by models with our "standard" (As04) mixture in which only few elements are varied each time by $\sim 20 \div 30 \%$, keeping the total metallicity constant. This could reflect not only an update of the solar mixture, but also a possible scatter in the composition of an open cluster: indeed, although stars in the same cluster are usually assumed to have the same chemical composition, an intrinsic dispersion in $[\mathrm{Fe} / \mathrm{H}]$ (up to $\sim \pm 0.15 \mathrm{dex}$ ) among cluster members cannot be excluded (e.g. Nissen 1988; Schuler et al. 2006). Analogous variations may exist for other elements. In Fig. 7 we show only the results for $1.0 M_{\odot}$, since the overall behavior was the same as in the previous calculations. The upper panel of Fig. 7 shows the effects on Li depletion when $\mathrm{C}$ and $\mathrm{O}$ are changed maintaining the sum unaltered in mass of the two elements $\left(X_{\mathrm{C}}+X_{\mathrm{O}}\right)$.

\footnotetext{
${ }^{3}$ http://www.t4.lanl.gov/cgi-bin/opacity/tops.pl
}
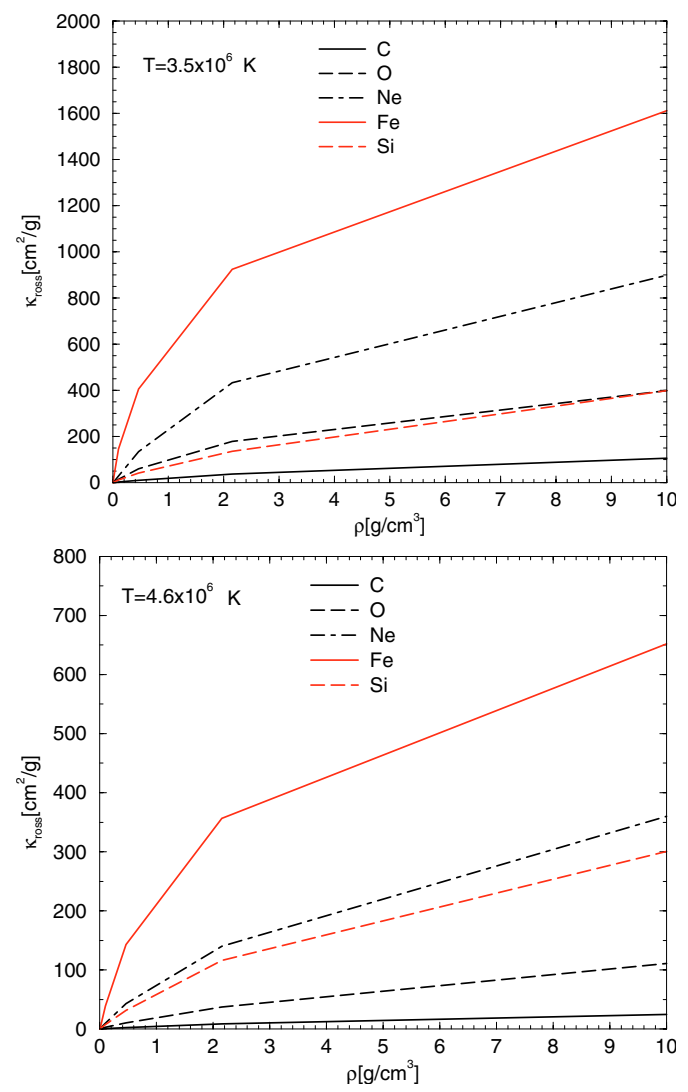

Fig. 5. The Rosseland opacity for $T=3.5 \times 10^{6} \mathrm{~K}$ (left panel) and $T=4.6 \times 10^{6} \mathrm{~K}$ (right panel) as a function of the density for several pure elements, as labeled. The opacity values are obtained from the URL http://www.t4.lanl.gov/cgi-bin/opacity/tops.pl.

In the first case $\mathrm{C}$ is decreased by $20 \%$ in mass while in the second case $\mathrm{O}$ is decreased by the same amount (models Nos. 10 and 11 in Table 1). An enhancement in $\mathrm{O}$ abundance increases the Li depletion, due to the higher opacity at the bottom of the convective zone and thus to the deeper extension of the convective envelope; the opposite happens when $\mathrm{O}$ is decreased. However, the effect is not symmetric: this is due to the fact that we keep the sum of the abundance in mass of the two elements - and thus the total metallicity - constant, and the two elements have different atomic weights and "standard" abundances; therefore, $\mathrm{C}$ and $\mathrm{O}$ are varied by different total amounts in number. These variations have different effects on the global mixture and on the opacity, and thus on Li depletion. The middle panel of Fig. 7 shows the results of a decrease/increase by $30 \%$ in mass of the $\mathrm{Ne}$ mass fraction (with a corresponding $\mathrm{O}$ variation to preserve $X_{\mathrm{O}}+X_{\mathrm{Ne}}$; models Nos. 12 and 13 in Table 1). Due to the higher $\mathrm{Ne}$ opacity, an increase/decrease of $\mathrm{Ne}$ abundance leads to a decrease/increase of the $\mathrm{Li}$ surface abundance but, in this case, the effect is obviously symmetric. The last numerical experiment (bottom panel of Fig. 7) evaluates the effects of increasing/decreasing the relative abundance of CNO elements and elements heavier than $\mathrm{O}$ (models Nos. 14 and 15 of Table 1): an increase in CNO with respect to heavier elements produces a lower depletion, and vice versa. The results of all the previous numerical experiments can be understood in terms of the radiative opacity of the heavy elements. In particular, from the point of view of Li depletion of low mass stars, the maximum depth reached by the convective envelope is very important, which in turn is sensitive to the opacity in the temperature range $3 \div 4 \times 10^{6} \mathrm{~K}$ (see Fig. 1). In this interval the most opaque 

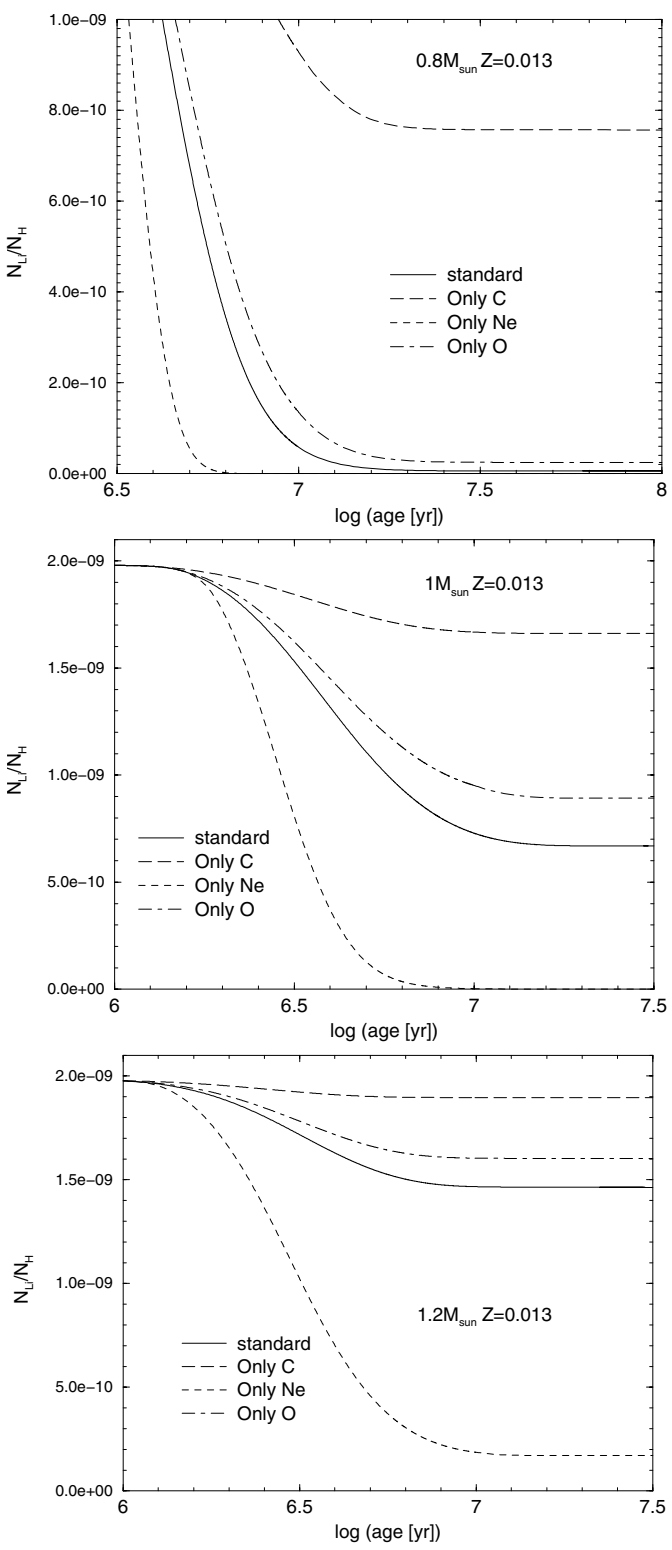

Fig. 6. Surface Li abundance as a function of time for PMS models of 0.8 (upper panel), 1.0 (middle panel) and 1.2 (lower panel) $M_{\odot}$. For each mass Li abundance is calculated for $Z=0.013, Y=0.27, \alpha=1.9$ for a metallicity due to only one element: $\mathrm{C}, \mathrm{O}$, and $\mathrm{Ne}$. The results are compared with the "standard" model, where the As04 mixture is adopted. Note the different scales adopted in the three panels.

elements, among those with a significant abundance, are the elements heavier than $\mathrm{O}$. If the abundance of these elements is higher, then the mixture is more opaque and thus the convective envelope is deeper. At fixed metallicity $(Z=0.013)$, the total abundance of the elements heavier than $\mathrm{O}$ increases progressively in the following mixtures: GN93, GS98, As05 and As04, thus explaining the behavior shown in Fig. 3.

\section{Models with fixed [Fe/H]}

In the previous sections we analysed the effects of a mixture variation at fixed global metallicity: however, the variation in the solar composition also affects the conversion of the $[\mathrm{Fe} / \mathrm{H}]$ value of the observed stars (derived through a spectroscopic analysis) to the total metallicity $Z$. This point is very subtle because to
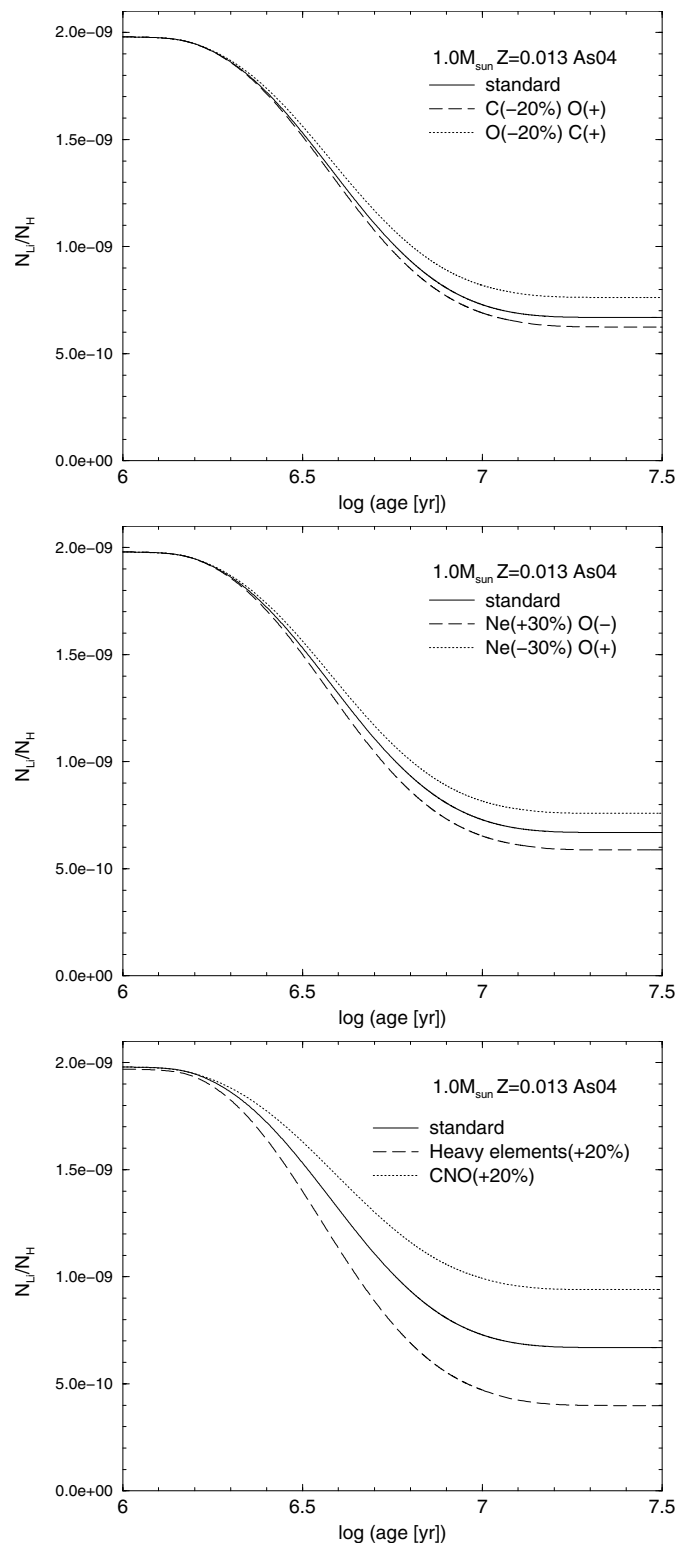

Fig. 7. $\mathrm{Li}$ abundance vs. time for PMS models of $1 M_{\odot}$. We adopted, as usual, $Z=0.013, Y=0.27$, and $\alpha=1.9$ and we varied the mass fraction of some elements starting from the As04 mixture (see text).

compute stellar models one needs both the Fe abundance and the total metallicity $Z$; the two quantities are related through the distribution of elemental abundances. In general, unless individual abundances are derived, a solar mixture is adopted. As a consequence, a revision of the photospheric solar abundance directly leads to a variation of the inferred total metallicity from the observed $[\mathrm{Fe} / \mathrm{H}]$. For solar-like stars, $[\mathrm{Fe} / \mathrm{H}]$ can be estimated by means of differential analysis, that is by comparison of the $\mathrm{Fe}$ lines of the star with those of the Sun. In such a case, the numerical value of $[\mathrm{Fe} / \mathrm{H}]$ is unaffected by a change of the solar mixture and the updated global metallicity of the star can be easily derived by adopting the new value of $(Z / X)_{\odot}$. That is:

$\log (Z / X)_{*}=[\mathrm{Fe} / \mathrm{H}]+\log (Z / X)_{\odot}$.

In order to give a quantitative estimate of the change of $Z$ (at fixed $[\mathrm{Fe} / \mathrm{H}]$ ) when the mixture and $Z / X_{\odot}$ are altered, for the GN93 composition $[\mathrm{Fe} / \mathrm{H}]=0$ corresponds to $Z \sim 0.018$, while for the As04 mixture, as already discussed, the corresponding metallicity is $Z \sim 0.013$ (standard value). Figure 8 shows the 


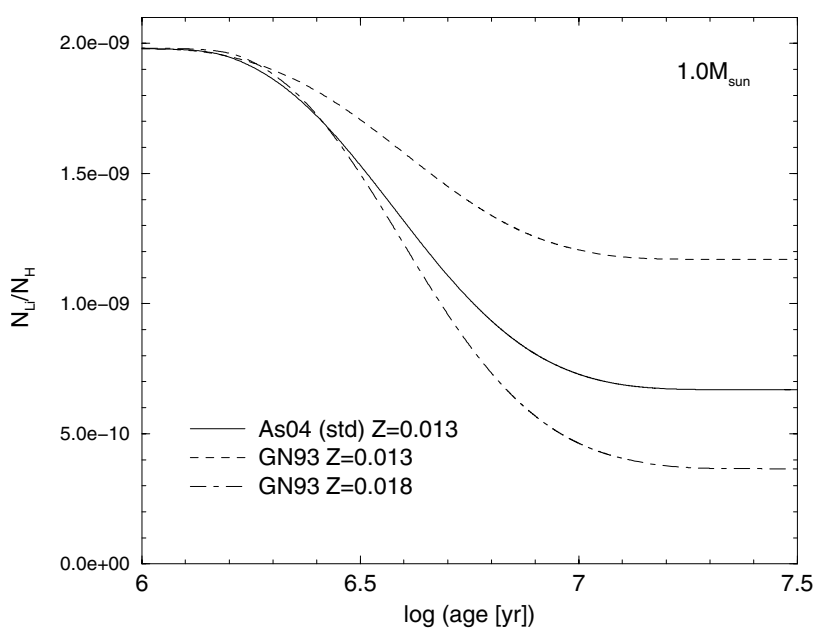

Fig. 8. Surface Li abundance vs. age for our standard model (As04 mixture with $Z=0.013$ ) compared to a model with $Z=0.013$ and GN93 mixture (the same of Figs. 2-4) and to a model with the GN93 solar composition and metallicity $Z=0.018$ (see text).

PMS Li depletion for a $1 M_{\odot} \operatorname{model}(Y=0.27, \alpha=1.9)$ for the GN93 composition and $Z=0.018$ (model No. 16 in Table 1) compared to our "standard" model (As04 mixture and $Z=0.013$ ); for reference, we also plot the model with GN93 mixture and $Z=0.013$, already reported in Fig. 3 . For the model with $Z=0.018$ the effect of an increase in the opacity - due to the increase of $Z$ - overcomes the influence of the mixture change.

We show in Fig. 9 a comparison between our "standard" model (As04 mixture), some of the models presented in Fig. 7 (all with $Z=0.013$ ), the GN93 model with $Z=0.018$, and the observational data for three young open clusters (ages $\sim 30 \div 50 \mathrm{Myr}$ ), in which solar-type stars are on the ZAMS. All the clusters plotted have $[\mathrm{Fe} / \mathrm{H}] \sim 0$ : IC 2602 (Randich et al. 2001), IC 2391 (Stauffer et al. 1989), and IC 4665 (Martín \& Montes 1997; see also Shen et al. 2005, for the estimate of the metallicity). The Li data for the clusters have been analysed by Sestito \& Randich (2005), starting from the equivalent widths published in the literature and using the same method of analysis. This plot compares the variations of our theoretical results - due to changes in the composition - and Li abundances observed in stars, and is not an attempt to fit the empirical data. For the reasons discussed in Sect. 1, even an apparent agreement between the theoretical predictions and the data of a cluster with a given age could be fictitious; to claim a real agreement between theory and observations, i.e. the goodness of the physical inputs and the macroscopic mechanisms adopted in the calculations, the models should be simultaneously compared to data from clusters with different chemical compositions and ages. The plot clearly shows how the match between theory and observations is strongly dependent on the adopted metallicity mixture and how the situation gets worse towards lower masses.

\section{Summary}

We presented an investigation of the effects of the chemical abundances on stellar opacity and ${ }^{7} \mathrm{Li}$ depletion. Within the "standard" scenario for stellar evolution - in which the position of the convective boundaries are determined following the Schwarzschild criterion - the theoretical predictions for the temporal behavior of surface $\mathrm{Li}$ depletion are affected by various uncertainties; among these a major role is played by radiative

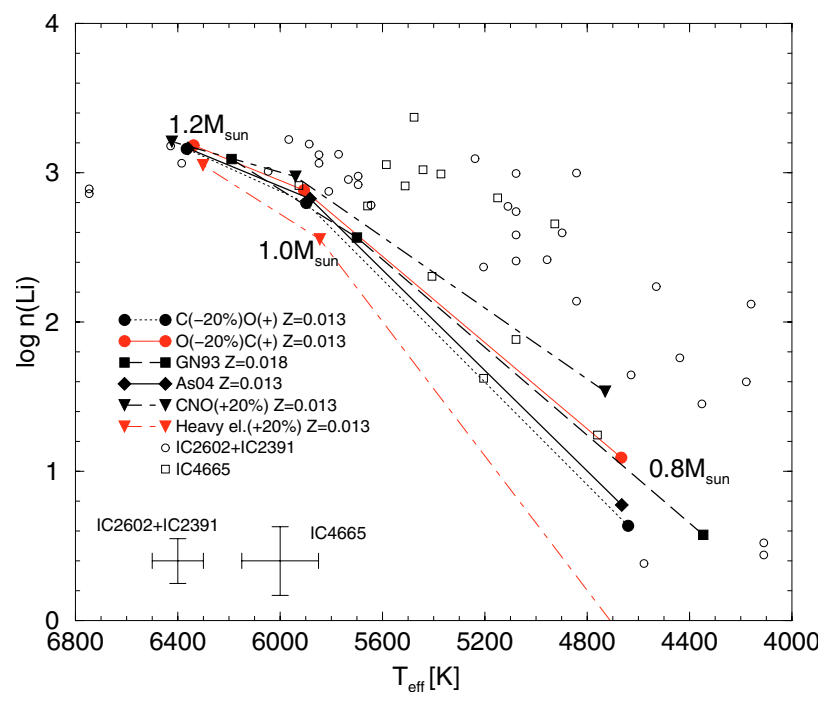

Fig. 9. Li abundance vs. $T_{\text {eff }}$ : comparison between the observational data for young open clusters (ages $\sim 30 \div 50 \mathrm{Myr}$ ) and the predictions of models. For the sake of clearness the calculations done for the three selected masses for each mixture are connected by lines. The mean observational error bars for the clusters are also shown.

opacity and therefore by the metallicity and the distribution of the single elements in the global mixture.

Focusing on pre-main sequence evolution, where the largest amount of Li burning is predicted by standard theories, we computed stellar models for three selected masses $(0.8,1.0$ and 1.2 $M_{\odot}$, with $\left.Z=0.013, Y=0.27, \alpha=1.9\right)$ by varying the global mixture (Grevesse \& Noels 1993; Grevesse \& Sauval 1998; Asplund et al. 2004; Asplund et al. 2005), showing that the effects of the chemical abundances must be taken into account. We have quantitatively computed the contribution of elements heavier than $\mathrm{O}$ for Pop. I models. The comparison between models with different mixtures revealed that, at fixed $Z$, a larger amount of $\mathrm{Li}$ is depleted in the presence of a larger fraction of elements heavier than $\mathrm{O}$. Therefore these species although less abundant than $\mathrm{CNO}$ - play a fundamental role in stellar opacity and PMS Li evolution.

These results have been interpreted by analysing the contribution of the single elements to the opacity at the temperatures and densities typical of the base of the convective envelope and the effects on Li depletion of single element variations in the mixture.

In addition, we computed models with the GN93 solar composition in which the global metallicity $Z$ is varied, showing that the effect of increasing $Z$ overcomes the influence of the mixture change. We conclude that changes in the chemical composition do affect Li depletion. Comparisons between model predictions and observations must take into account the element distribution in the stars. Moreover, the abundance determinations for open clusters should rely on the 3D atmospheric models which are more consistent than the usually adopted 1D models. None of the adopted solar mixtures and variations in the chemical composition appear to explain the very small amount of PMS Li depletion observed for solar-type stars.

Acknowledgements. We are extremely grateful to V. Castellani and S. Shore for a careful reading of the manuscript. We warmly thank the referee (A. Weiss) for the useful comments. Financial support for this work was provided by the Ministero dell'Istruzione, dell'Università e della Ricerca (MIUR) under the scientific project "Continuity and discontinuity in the Galaxy formation" (P.I.: R. Gratton). 


\section{References}

Alexander, D. R., \& Ferguson, J. W. 1994, ApJ, 437, 879

Asplund, M., Grevesse, N., Sauval, A. J., Allende Prieto, C., \& Kiselman, D. 2004, A\&A, 417, 751

Asplund, M., Grevesse, N., \& Sauval, A. J. 2005, in Cosmic Abundances as Records of Stellar Evolution and Nucleosynthesis, ed. F. N. Bash, \& T. J. Barnes, ASP Conf. Ser., 336, 25

Bahcall, J. N., \& Serenelli, A. M. 2005, ApJ, 626, 530

Bensby, T., Feltzing, S., \& Lundström, I. 2003, A\&A, 410, 527

Cariulo, P., Degl'Innocenti, S., \& Castellani, V. 2004, A\&A, 424, 927

Carretta, E., Bragaglia, A., Gratton, R., \& Tosi, M. 2004, A\&A, 422, 951

Castellani, V., Degl'Innocenti, S., \& Marconi, M. 1999, MNRAS, 303, 265

Chen, Y. Q., Nissen, P. E., Zhao, G., Zhang, H. W., \& Benoni, T. 2000, A\&AS, 141,491

Chieffi, A., \& Straniero, O. 1989, ApJS, 71, 47

Ciacio, F., Degl'Innocenti, S., \& Ricci, B. 1997, A\&AS, 123, 449

D’Antona, F., \& Mazzitelli, I. 1997, MmSAIt, 68, 807

Friel, E., Janes, K. A., Tavarez, M., et al. 2002, AJ, 124, 2693

Grevesse, N. 1991, A\&A, 242, 488

Grevesse, N., \& Anders, E. 1991, in Solar Interior and Atmosphere (Tucson, AZ, University of Arizona Press), 1227

Grevesse, N., \& Noels, A. 1993 in Origin and Evolution of the Elements, ed. N. Prantzos, E. Vangioni-Flam, \& M. Cassé (Cambridge: Cambridge University Press), 15

Grevesse, N., \& Sauval, A. J. 1998, SSRv, 85, 161

Iglesias, C., \& Rogers, F. J. 1996, ApJ, 464, 943

Jeffries, R. D. 2000 in Stellar Clusters and Associations: Convection, Rotation, and Dynamos, ed. R. Pallavicini, G. Micela, \& S. Sciortino, ASP Conf. Ser., 198,245

Jeffries, R. D. 2006, in Chemical Abundances and Mixing in Stars in the Milky Way and its Satellites, ed. L. Pasquini, \& S. Randich, ESO Astrophys. Symp., in press

Luridiana, V., Peimbert, A., Peimbert, M., \& Cerviño, M. 2003, ApJ, 592, 846

Martín, E., \& Montes, D. 1997, A\&A, 318, 805

Nissen, P. 1988, A\&A, 199, 146

Nordström, B., Mayor, M., Andersen, J., et al. 2004, A\&A, 418, 989
Olive, K. A., \& Skillman, E. D. 2004, ApJ, 617, 29

Pagel, B. E. J., \& Portinari, L. 1998, MNRAS, 298, 747

Palla, F. 2002 in Star Formation and the Physics of Young Stars, ed. Bouvier, \& J.-P. Zahn, EDP Sciences (Aussois, France: EAS Publications Series), 3, 111

Piau, L. 2005, ApJ, submitted [arXiv: astro-ph/0511402]

Piau, L., \& Turck-Chièze, S. 2002, ApJ, 566, 419

Piau, L., Randich, S., \& Palla, F. 2003, A\&A, 408, 1037

Pinsonneault, M. 1997, ARA\&A, 35, 557

Potekhin, A. Y. 1999, A\&A, 351, 787

Randich, S. 2006, in Chemical Abundances and Mixing in Stars in the Milky Way and its Satellites, ed. L. Pasquini, \& S. Randich, ESO Astrophys. Symp., in press

Randich, S., Pallavicini, R., Meola, G., Stauffer, J. R., \& Balachandran, S. C. 2001, A\&A, 372, 862

Randich, S., Sestito, P., Primas, F., Pasquini, L., \& Pallavicini, R. 2006, A\&A, 450,557

Rogers, F. J. 2001, Contributions to plasma physics, V41(N2-3), 179

Schuler, S. C., Hatzes, A. P., King, J. R., Kuerster, M., \& The, L.-S. 2006, AJ, 131,1057

Sestito, P., \& Randich, S. 2005, A\&A, 442, 615

Sestito, P., Randich, S., Mermilliod, J.-C., \& Pallavicini, R. 2003, A\&A, 407, 289

Shen, Z.-X., Jones, B., Lin, D. N. C., Liu, X.-W., \& Li, S.-L. 2005, ApJ, 635, 608

Siess, L., Dufour, M., \& Forestini, M. 2000, A\&A, 358, 593

Stahler, S. W., \& Palla, F. 2004, The Formation of Stars (New York: Wiley)

Stauffer, J., Hartmann, L. W., Jones, B. F., \& McNamara, B. R. 1989, ApJ, 342, 285

Swenson, F. J., Faulkner, J., Iglesias, C. A., Rogers, F. J., \& Alexander, D. R. 1994a, ApJ, 422, L79

Swenson, F. J., Faulkner, J., Rogers, F. J., \& Iglesias, C. A. 1994b, ApJ, 425, 286

Thoul, A. A., Bahcall, J. N., \& Loeb, A. 1994, ApJ, 421, 828

Turcotte, S., \& Christensen-Dalsgaard, J. 1998, Space Sci. Rev., 85, 133

Turcotte, S., Richer, J., \& Michaud, G. 1998a, ApJ, 504, 559

Turcotte, S., Richer, J., Michaud, G., Iglesias, C., \& Rogers, F. 1998b, ApJ, 504, 539 